\title{
The Role of SOFA Score in Survival COVID-19 Patients Admitted to Intensive Care Unit
}

\author{
Matyn Pernama ${ }^{a}$, Bambang Pujo Semedi ${ }^{\text {b,d* }}$, Kuntaman Kuntaman ${ }^{c, d}$ \\ a Study Program of Specialist in Anasthesiology and Intesive Care, Department of Anasthesiology and \\ Reanimasi, Faculty of Medicine Universitas Airlangga \\ ${ }^{\mathrm{b}}$ Department of Anasthesiology and Reanimasi, Faculty of Medicine Universitas Airlangga \\ ${ }^{c}$ Department of Medical Microbiology, Faculty of Medicine Universitas Airlangga \\ ${ }^{d}$ Dr. Soetomo General Academic Hospital, Surabaya, Indonesia
}

\begin{abstract}
The novel coronavirus disease, COVID-19, is caused by a SARS-COV-2 infection and manifested in some clinical forms from mild to critical disease. The Sequential Organ Failure Assessment (SOFA) score has been used to predict the clinical outcomes of patients admitted to intensive care units with multiple organ failures. This study aimed to analyse the cut-off of the SOFA score with the best accuracy in predicting mortality in severe and critical COVID-19 population, and compared the survival rate difference of the high and low SOFA score according to the cut-off with the best accuracy from our study. This retrospective observational study analysed all of the severe and critical COVID-19 patients admitted to DR.Soetomo Hospital, Surabaya, Indonesia, from June to September 2020. We excluded patients who had died less than 48 hours upon ICU admission and patients who were discharged against medical advice (DAMA). A total of 123 patients were eligible. SOFA score significantly predicted ICU mortality of COVID-19 patients with the Area-under-curve of $0.9(p<0.001)$. The survival rate of patients with the SOFA score of $\leq 7$ was $90 \%$, compared to patients with the SOFA score of $>7$ with a survival rate of $10 \%$. Patients with SOFA score $\leq 7$ had a significantly longer survival time during ICU care $(\mathrm{HR}=15.85$, $\mathrm{p}<0.001)$. Our study proved that patients with higher SOFA scores had a significantly different survival outcome during the ICU stay, with the score cut-off of 7 . This score cut-off could significantly discriminate ICU survival with high predictive values.
\end{abstract}

Keywords: COVID-19; Survival; SOFA score

\section{Introduction}

A novel coronavirus disease, termed COVID-19, was caused by SARS-COV-2 that had a virulence to infect ciliated cells and secretory cells of respiratory epithelium simultaneously and could manifest as severe acute respiratory syndrome.(1) This RNA virus was first identified in year 2019 in Wuhan, China, and has been established as a Public Health Emergency of International Concern (PHEIC) by the World Health Organization on January 2020.(2) As of December 7, 2021, 265 million cases has been confirmed, and as many as 5,254,116 people has been confirmed to passed away because of COVID-19.(3) A study from Baud et al., explained the real estimate of COVID-19 with severe illness. Approximately, 16\% of severe COVID-19 following hospitalization, $5 \%$ of these patients were admitted to ICU, $2.3 \%$ required intubation, and $1.4 \%$ passed away.(4)

SARS-COV-2 infection causes interstitial inflammation and subsequent lung injury. Activation of Angiotensin II pathway influence the adaptive immunity by stimulating macrophages ang the other immune cells, which further caused acute respiratory distress syndrome in the critical cases. The inductions of immune cells lead to their propagation and their production of pro-inflammatory cytokines such as IL-6, and tumour necrosis factor-alpha $(\mathrm{TNF}-\alpha)$. Activation of dendritic cells as the antigen presenting cells and also macrophage, enhance the phagocytosis and apoptosis of virus-infected host cells. The enhanced levels of pro-inflammatory mediators followed by cytokine storm and hyperinflammation syndrome, that worsen the clinical manifestations of COVID19. Cytokine storm, that is followed by the attacking immune system via SARS-CoV2, results in lung failure, multiple-organ dysfunction, and mortality in severe cases.(5)

Various prognostic scoring had been developed to predict the survival of severe and critical COVID-19 patients and thus could improve effective treatment strategies and evaluate the disease progression further. The Sequential Organ Failure Assessment (SOFA) score has been used to predict the clinical outcomes of patients admitted to

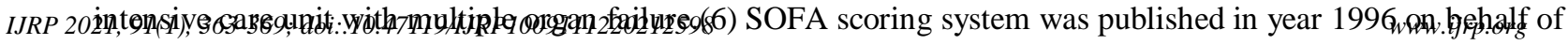
Working Group on Sepsis-Related Problems of the European Society of Intensive Care Medicine. SOFA score 
performance was based on the 6 major organ functions: circulation, respiration, liver, renal function, central nervous system, and coagulation function. Each organ system had a ranging score from 0 to 4 . SOFA score is a practical tool to evaluate organ function during ICU stay.(7) These scores have also demonstrated utility in evaluating disease severity outside of the ICU setting. SOFA score at the time of emergency department presentation predicts outcomes in severe sepsis.(8)

Therefore, we analysed the cut-off of the SOFA score with the best accuracy in predicting mortality in severe and critical COVID-19 population, and compared the survival rate difference of the high and low SOFA score according to the cut-off with the best accuracy from our study.

\section{Methods}

\subsection{Study design}

This retrospective cohort study was conducted retrospectively among severe and critical COVID-19 patients admitted to ICU from June 2020 to September 2020 in Dr Soetomo General Hospital, Surabaya, Indonesia. Patients were followed-up from the early ICU admission until ICU discharge. SOFA score was calculated several times during the ICU follow-up. The highest and lowest SOFA scores were documented during ICU care.

Study population

We included all adult patients with the minimum age of 18 years old who were confirmed for COVID-19 and with severe and critical illness manifestation who were admitted to COVID-19 ICU. Used a total sampling for this study. We excluded patients who had died less than 48 hours upon ICU admission, and patients who were discharged against medical advice (DAMA).

\subsection{Measurements}

Study personnel reviewed electronic health records according to the CRF (case record form) in ICU, to collect baseline patient characteristics including age, gender, body mass index, medications, present and past medical histories, comorbidities, and disease progression during ICU stay. COVID-19 was confirmed by SARS-CoV-2 testing using polymerase chain reaction (PCR) method. Severe COVID-19 pneumonia used the criteria of the following: clinical symptoms of pneumonia (fever, cough, shortness of breath, tachypnoea), with one of the following: 1). respiratory rates of $>30$ times/minutes; 2). severe respiratory distress, or 3). $\mathrm{SpO} 2$ of $<90 \%$ in room air. Critical COVID-19 patients were the patients with presentation of ARDS, or sepsis, or septic shock. Sepsis and septic shock were defined according to Sepsis bundle guideline 2021.(9) Septic shock has a manifestation of sepsis symptoms, accompanied by the persistent hypotension which require vasopressor to maintain mean arterial pressure for the minimum of $65 \mathrm{mmHg}$, with lactate serum concentration $>2 \mathrm{mmol} / \mathrm{L}$ $(18 \mathrm{mg} / \mathrm{dL})$ despite of adequate fluid resuscitation.

The maximum SOFA score describes the highest daily SOFA score over the course of the study period. Calculation of SOFA score based on six criteria reflecting the function of an organ system (respiratory, cardiovascular, renal, neurological, hepatic and haematological) and allocates a score of $0-4$ as elaborated below in Table 1, adapted from Vincent et al.(10).

Table 1. Sequential Organ Failure Assessment (SOFA) Score Criteria

\begin{tabular}{lc}
\hline Organ systems & Scores \\
\hline $\mathrm{Respiratory} \mathrm{system} \mathrm{PaO}_{2} / \mathrm{FiO}_{2}(\mathrm{mmHg})$ & \\
$>400$ & 0 \\
$<400$ & 1 \\
$<300$ & 2 \\
$<200$ with respiratory support & 3 \\
Nervous system & \\
Glasgow comma score & 0 \\
15 & 1 \\
$13-14$ & 2 \\
$10-12$ & 3 \\
$6-9$ & 4 \\
$\quad 6$ & 4 \\
Cardiovascular system & \\
Mean Arterial pressure & \\
MAP $>70 \mathrm{mmHg}$ & 0
\end{tabular}




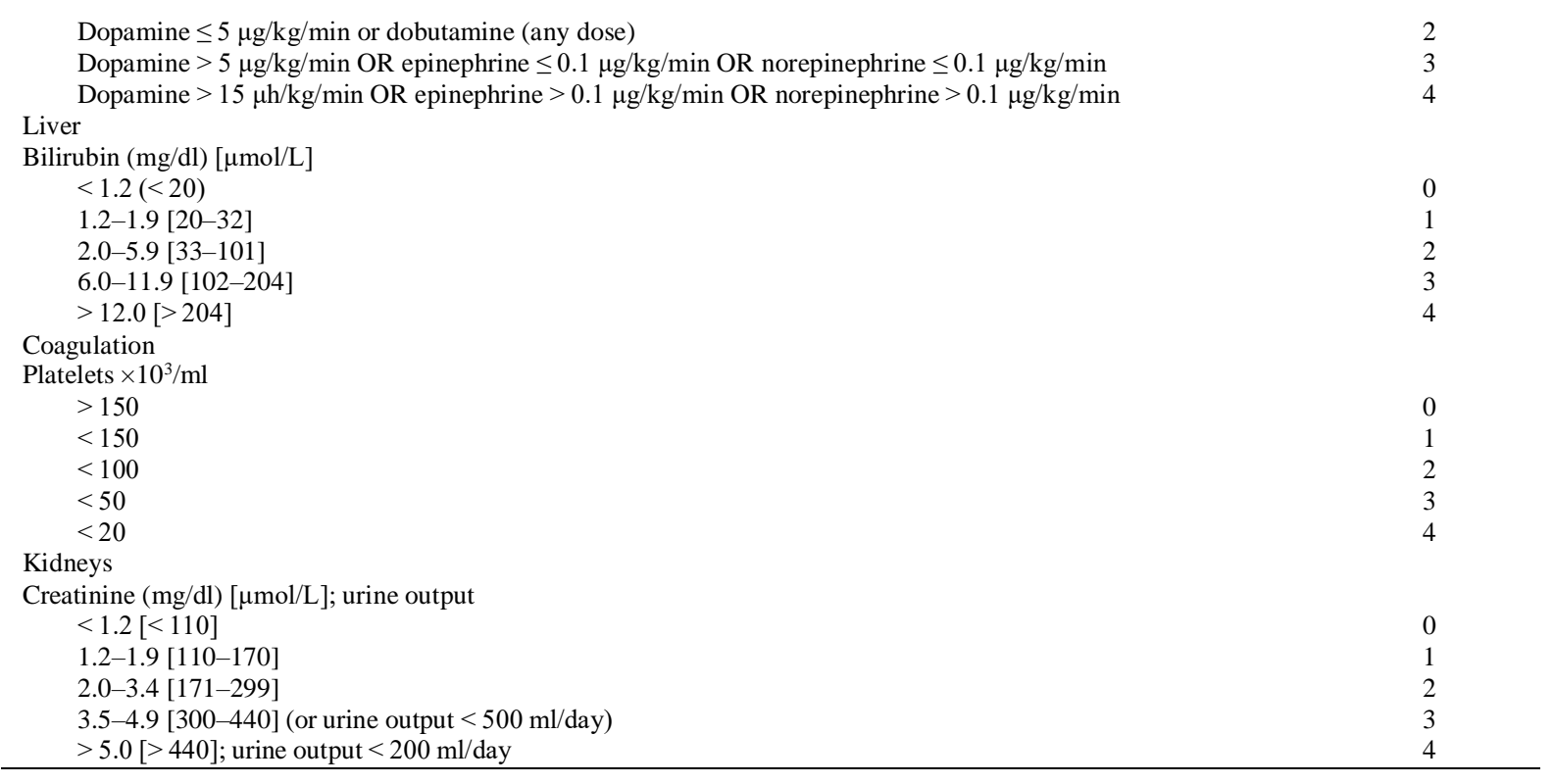

Survival criteria were the severe and critical COVID-19 patients who were alive until the ICU discharge.

\subsection{Statistical analysis}

Continuous variables were reported as median and interquartile range (IQR). Categorical variables were reported as frequencies and proportions. Bivariate analysis was made with the Mann-Whitney for continuous variables and the Fishers exact test for categorical variables. A two-sided $\mathrm{P}$ value $<0.05$ was used to indicate statistical significance without adjustment for multiple testing. Receiver operating characteristic (ROC) curve's area under the curve (AUC) used the significance of $95 \%$ and cut-off $>0.7$ was considered to have a strong association. Sensitivity and specificity were calculated with the aid of Ms. Excel. Survival analysis used KaplanMeier curve and cox-regression. Statistical analysis was calculated and presented by using SPSS version 21.

\section{Results}

A total of 134 patients met the inclusion criteria and 11 patients were excluded, therefore 123 patients were eligible in this study. Baseline characteristics of the subjects shows in Table 2. Median age of the severe and critical COVID patients was 50 years old. Body mass index was significantly different in the patients with high SOFA score (>7) compared to patients with the maximum SOFA score not more than 7. Patients with higher SOFA score tend to have lower MAP, higher leukocyte counts, and higher concentration of sepsis biomarkers (lactate and procalcitonin) . Acute Kidney Injury (AKI) was the complication that was more commonly found in patients with the higher SOFA score.

Table 2. Baseline characteristics

\begin{tabular}{|c|c|c|c|c|}
\hline Variables* & $\begin{array}{l}\text { All patients } \\
\mathrm{N}=123\end{array}$ & $\begin{array}{c}\text { SOFA score }>7 \\
n=67(54.5 \%)\end{array}$ & $\begin{array}{c}\text { SOFA } \leq 7 \\
\mathrm{~N}=56(45.5 \%)\end{array}$ & P-value \\
\hline Age & $50.0 \pm 12.0$ & $51 \pm 12.2$ & $48.5 \pm 12.3$ & 0.464 \\
\hline Male Gender & $78(63.4 \%)$ & $45(67.1 \%)$ & $33(58.9 \%)$ & 0.355 \\
\hline Survive & $62(50.4 \%)$ & $11(16.4 \%)$ & $51(91.0 \%)$ & $<0.001$ \\
\hline Body Mass Index & $26.1 \pm 5.6$ & $27.1 \pm 6.4$ & $25.7 \pm 4.1$ & 0.018 \\
\hline Length of stay (days) & $8.0 \pm 5.1$ & $9 \pm 5.9$ & $6.5 \pm 3.3$ & 0.010 \\
\hline \multicolumn{5}{|l|}{ Vital signs } \\
\hline Systolic Blood Pressure (mmHg) & $100.0 \pm 19.4$ & $90 \pm 18.9$ & $110 \pm 12.6$ & $<0.001$ \\
\hline Diastolic Blood Pressure $(\mathrm{mmHg})$ & $58.0 \pm 12.5$ & $50 \pm 11.1$ & $60 \pm 10.6$ & $<0.001$ \\
\hline Mean Arterial Pressure & $70.0 \pm 14.1$ & $63 \pm 12.5$ & $76 \pm 11.2$ & $<0.001$ \\
\hline Body Temperature & $37.6 \pm 3.1$ & $38 \pm 4.3$ & $37.2 \pm 0.5$ & $<0.001$ \\
\hline \multicolumn{5}{|l|}{ Laboratory results } \\
\hline Haemoglobin & $11.5 \pm 2.1$ & $10.7 \pm 2.2$ & $12.3 \pm 1.9$ & 0.023 \\
\hline Haematocrit & $35.0 \pm 6.3$ & $33 \pm 6.7$ & $37 \pm 5.4$ & 0.010 \\
\hline Thrombocyte & $250000 \pm 255529$ & $239500 \pm 329263$ & $258000 \pm 120392$ & 0.475 \\
\hline Leucocyte & $19900 \pm 12118$ & $23470 \pm 12165$ & $14015 \pm 10026$ & $<0.001$ \\
\hline
\end{tabular}




\begin{tabular}{|c|c|c|c|c|}
\hline Lactate & $1.7 \pm 2.7$ & $2.2 \pm 3.5$ & $1.1 \pm 0.9$ & $<0.001$ \\
\hline SGOT & $69.0 \pm 136.8$ & $73 \pm 164.2$ & $56.5 \pm 94$ & 0.034 \\
\hline SGPT & $78.0 \pm 74.4$ & $77 \pm 74.4$ & $83.5 \pm 75.1$ & 0.839 \\
\hline Blood Urea Nitrogen & $34.0 \pm 45.8$ & $61 \pm 50.0$ & $18.0 \pm 14.5$ & $<0.001$ \\
\hline Creatinine Serum & $1.3 \pm 9.1$ & $3.5 \pm 11.9$ & $0.9 \pm 1.3$ & $<0.001$ \\
\hline C-Reactive Protein & $8.9 \pm 2.9$ & $11.9 \pm 38.9$ & $6.4 \pm 9.3$ & 0.083 \\
\hline Procalcitonin & $0.7 \pm 2.3$ & $2.0 \pm 28.7$ & $0.3 \pm 13.5$ & $<0.001$ \\
\hline Blood sugar & $234 \pm 121.8$ & $267 \pm 132.5$ & $198 \pm 88.6$ & 0.005 \\
\hline HBA1C & $6.4 \pm 2.2$ & $6.5 \pm 2.5$ & $6.0 \pm 1.6$ & 0.031 \\
\hline D-dimer & $3260 \pm 7836$ & $3650 \pm 7193$ & $2875 \pm 8609$ & 0.166 \\
\hline Ferritin & $1045 \pm 1870$ & $1220 \pm 2407$ & $825 \pm 819$ & 0.148 \\
\hline PTT & $13.5 \pm 4.3$ & $14.8 \pm 5.0$ & $12.8 \pm 2.9$ & 0.016 \\
\hline APTT & $33.3 \pm 29.1$ & $38.6 \pm 32.1$ & $30.0 \pm 22.9$ & 0.001 \\
\hline Bilirubin Direct & $0.7 \pm 4.3$ & $1.1 \pm 2.0$ & $0.5 \pm 6.0$ & $<0.001$ \\
\hline Bilirubin Total & $0.9 \pm 3.0$ & $1.4 \pm 4.0$ & $0.8 \pm 0.8$ & 0.001 \\
\hline Albumin & $2.8 \pm 0.3$ & $2.7 \pm 0.3$ & $3.0 \pm 0.3$ & $<0.001$ \\
\hline \multicolumn{5}{|l|}{ Respiratory } \\
\hline $\mathrm{P} / \mathrm{F}$ ratio & $94.0 \pm 64.8$ & $80.2 \pm 32.3$ & $136 \pm 77.0$ & $<0.001$ \\
\hline $\mathrm{SPO}_{2}$ & $94.0 \pm 5.1$ & $92 \pm 5.8$ & $95.5 \pm 3.2$ & $<0.001$ \\
\hline Ventilator support & $89(72.4 \%)$ & $67(100 \%)$ & $22(39.2 \%)$ & $<0.001$ \\
\hline High Flow Nasal Cannula & $48(39 \%)$ & $18(32.1 \%)$ & $30(53.6 \%)$ & 0.003 \\
\hline \multicolumn{5}{|l|}{ Comorbidities } \\
\hline Hypertension & $49(39.8 \%)$ & $31(46.3 \%)$ & $18(32.1 \%)$ & 0.140 \\
\hline Diabetes Mellitus & $59(48 \%)$ & $37(55.2 \%)$ & $22(39.3 \%)$ & 0.103 \\
\hline Tuberculosis & $1(0.8 \%)$ & $1(1.5 \%)$ & $0(0 \%)$ & 1.000 \\
\hline Chronic Kidney Disease & $5(4.1 \%)$ & $4(6.0 \%)$ & $1(1.7 \%)$ & 0.375 \\
\hline Asthma & $4(3.3 \%)$ & $2(3.0 \%)$ & $4(3.5 \%)$ & 1.000 \\
\hline Hepatitis & $18(14.6 \%)$ & $11(16.4 \%)$ & $7(12.5 \%)$ & 0.615 \\
\hline Acute Kidney Disease & $40(32.5 \%)$ & $36(53.7 \%)$ & $4(7.1 \%)$ & $<0.001$ \\
\hline \multicolumn{5}{|l|}{ Medications } \\
\hline Dopamine & $2(1.6 \%)$ & $2(3.0 \%)$ & $0(0 \%)$ & 0.295 \\
\hline Dobutamine & $21(17.1 \%)$ & $19(28.3 \%)$ & $2(3.5 \%)$ & $<0.001$ \\
\hline Norepinephrine & $54(43.9 \%)$ & $51(76.1 \%)$ & $3(5.3 \%)$ & $<0.001$ \\
\hline Epinephrine & $27(22 \%)$ & $26(38.8 \%)$ & $1(1.7 \%)$ & $<0.001$ \\
\hline Vasopressin & $22(17.9 \%)$ & $22(32.8 \%)$ & $0(0 \%)$ & $<0.001$ \\
\hline Antibiotics & $94(95.5 \%)$ & $30(53.6 \%)$ & $30(31.9 \%)$ & $<0.001$ \\
\hline Antiviral & $45(78.9 \%)$ & $43(76.8 \%)$ & $43(48.9 \%)$ & 0.316 \\
\hline Antifungal & $18(26.9 \%)$ & $5(8.9 \%)$ & $5(21.7 \%)$ & 0.012 \\
\hline
\end{tabular}

SOFA score significantly predicted ICU mortality of COVID-19 patients with the Area under curve of 0.9 $(\mathrm{p}<0.001)$. (Figure 1)

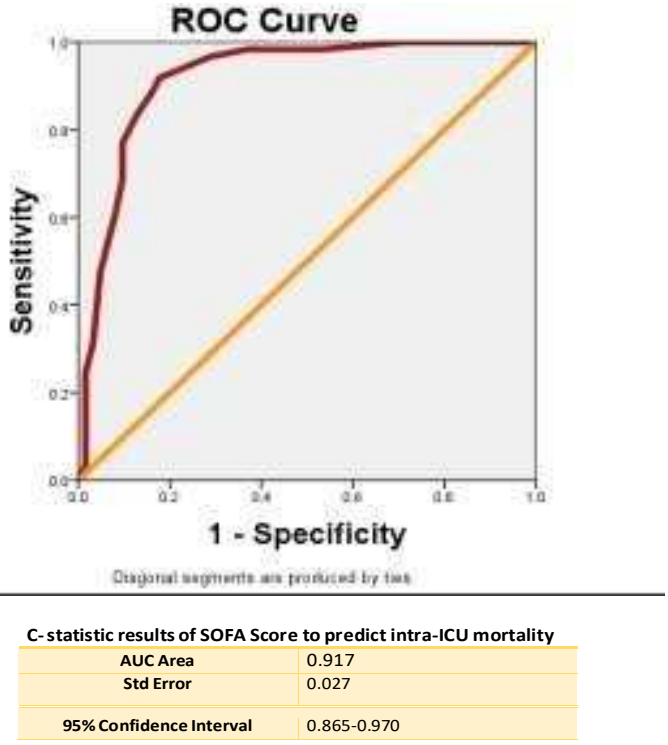

Fig. 1. SOFA score performance in predicting ICU mortality 
Survival rate of patients with the SOFA score of $\leq 7$ was $90 \%$, compared to patients with the SOFA score of $>7$ with survival rate of $10 \%$. Patients with SOFA score $\leq 7$ had a significantly longer survival time during ICU care $(\mathrm{HR}=15.85, \mathrm{p}<0.001)$. (Figure 2)

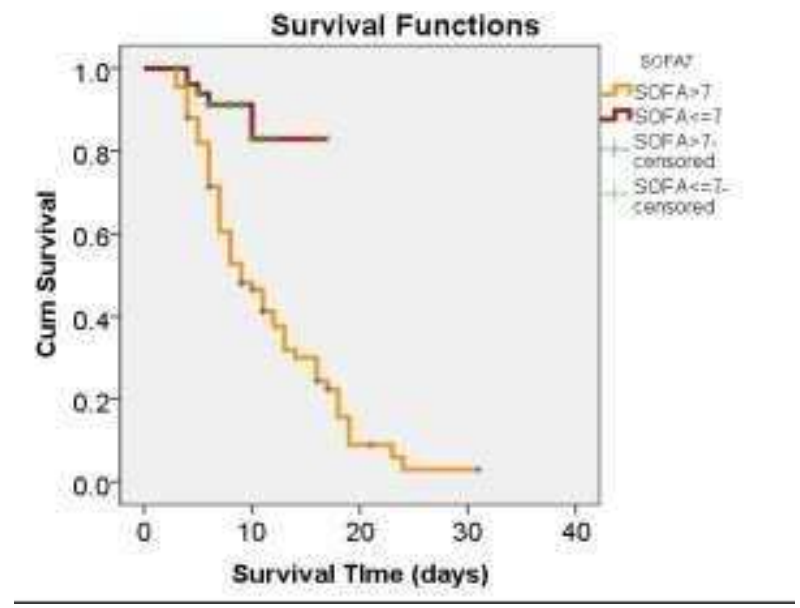

Fig. 2. Kaplan-Meier Curve of Survival among severe and critical COVID-19 patients

\section{Discussion}

Severe and critical COVID-19 could lead to and manifested as acute respiratory distress syndrome (ARDS), sepsis, and subsequent septic shock. SARS-COV-2 infection with severe to critical manifestation are able to cause multiple organ dysfunctions, such as acute myocardial injury, acute kidney injury and even to mortality.(11) SOFA score is simple and discriminate tool to measure both severity and number of multiple organ dysfunctions that also are the complications found in severe and critical COVID-19 patients. It was originally developed to assess the severity of organ dysfunction in severe sepsis, and it had been validated by many studies in ICU settings.(12)

Similar result was reconfirmed in our study that in severe and critical COVID-19 patients, SOFA score had a strong association with ICU mortality (AUC $=0.917, \mathrm{p}<0.001$ ). SOFA score cut-off that interfered the survival outcome of the patients was calculated; sensitivity and specificity of SOFA score with the cut-off of 7 were 0.918 and 0.823 , respectively. PPV was 0,84 and NPV 0.91. As much as $84 \%$ of patients with SOFA score of $>7$, were mortality during ICU stay in the population of severe and critical COVID-19 patients, with $8.9 \%$ of false negative result. SOFA score of $>7$ had a high sensitivity towards ICU mortality. These results were reconfirmed by the survival analysis in our study, with the Hazard ratio (Log Mantel Cox) of 15.85, and significancy of $<0.001$. The occurrence of ICU mortality in severe and critical COVID-19 patients during median interval time of 10.5 days was 15.85 times higher in patients with the SOFA score >7.(13) Survival rate of patients with SOFA score of $>7$ was only $10 \%$ with the median survival time of 9 days.

The virulence of SARS-COV-2 to cause multiorgan dysfunctions was hypothesized because of its binding receptors that could be found in many tissue types in human body. In the early infection, this virus targeted cells in respiratory tracts through the $\mathrm{S}$ protein binding to the Angiotensin converting enzyme 2 (ACE2) receptor.(14) ACE2 reduces angiotensin II by cleaving it to the sorter angiotensin(1-7) peptide, which can then activate the vasodilation-promoting and anti-inflammatory, therefore the binding of SARS-COV-2 spike protein to ACE2 receptor would leads to the opposite effects (pro-inflammatory).(15)

The following phase of SARS-COV-2 infection was the virus replication, as the replication accelerated, pulmonary capillary endothelial cells and epithelial-endothelial barriers' integrity were markedly affected and 
together with the advancing of the inflammation causing the infiltration of monocytes and neutrophils. Subsequently, thickening of the alveolar wall infiltrating airspaces worsen the endothelialitis. The alveolar spaces were filled with hyaline membrane formation in the pulmonary oedema settings, therefore the early stage of ARDS began.(16) Moreover, decrease of ACE2 expression and locally increased of angiotensin II production induce the pulmonary blood vessels leakage.

Continuous SARS-COV-2 infection with the sustainable capillary leakage consequences in its bloodstream spreading, called viremia. Viremia and the increase of ACE/Angiotensin signalling through AT1 receptor cause the further diminishment of ACE2 and therefore leads to the overactivation of pro-inflammatory cells and mediators, termed as cytokine storm.(17)

ACE2 expression was observed across more than 150 different cell types and mainly found in: enterocytes, renal tubules, gallbladder, cardiomyocytes, male reproductive cells, placental trophoblasts, ductal cells, eye, and vasculature.(18) A prior study also correlated the ARDS with the obstructive thrombo-inflammatory syndrome in the microvascular of COVID-19 patients' lung.(19) Reduction of ACE2 in many cell sites compromised the multiorgan damage directly, and indirectly by the cytokine storm. As a platelet production site, lung serves as the hematopoietic function and as the reservoir of megakaryocytes and hematopoietic progenitors, causing thrombocytopenia in COVID-19 case.(20) Another haematological abnormality in COVID-19 was the imbalance of coagulation pattern caused by the endotheliopathy. Relating to kidney injury caused by COVID-19, collapsing focal segmental glomerulosclerosis and podocyte damage were identified. An indirect mechanisms to renal damage were the tissue hypoxia, haemodynamic instability, sepsis, cytokine storm, and rhabdomyolysis.(21) COVID-19 manifestation inside the liver tissue were the infiltration of lymphocytes in sinusoidal spaces, hepatic necrosis including inside the portal vein, and also the Kupffer cells activation by the necrotic debris.(5) COVID19 also could affect nervous system directly and indirectly. COVID-19 directly affects ACE2 receptors in nuclei of brainstem as the cardiorespiratory regulator and indirectly by the loss of permeability in blood brain barrier caused by the endotheliopathy, therefore enhance the central nervous system infection.(22)

COVID-19 infection affected the 6 main organs function directly to the tissue injury and indirectly by the cytokine-storm's vicious cycle, parallel with the SOFA score that measures the function of these 6 main organs related to COVID-19 pathogenesis. Beyond this reason, our study proved that patients with higher SOFA score had a significantly different survival outcomes during the ICU stay, with the score cut-off of 7 . This score cut-off could significantly discriminate ICU survival with high predictive values.

\section{Conclusion}

Survival rate of COVID-19 patients admitted to ICU was significantly higher in patients with SOFA score of $\leq 7$, compared to the ones with SOFA score $>7$. Survival time was significantly shorter in patients whose SOFA score was $>7$. SOFA score could be used to discriminate the survival probability in severe and critical COVID19 patients admitted to ICU, regardless of their comorbidities. Further researches with bigger sample size are recommended to confirm our study results with the SOFA score cut-off of 7 for COVID-19 patients that were taken care in ICU.

\section{Conflict of Interest}

The authors declared no potential conflict of interest relevant to this article.

\section{Acknowledgement}

We are grateful for the support of COVID-19 ICU team in Dr. Soetomo General Hospital, Surabaya

\section{Authors' Agreement}

All authors gave final approval and agree to be accountable for all aspects of work, ensuring integrity and accuracy. 


\section{References}

1. Zhu N, Wang W, Liu Z, Liang C, Wang W, Ye F, et al. Morphogenesis and cytopathic effect of SARS-CoV-2 infection in human airway epithelial cells. Nat Commun. 2020 Aug;11(1):3910.

2. World Health Organization. International Guidelines for Certification and Classification (Coding) of Covid-19 as Cause of Death. Who [Internet]. 2020;(April):14. Available from: https://www.who.int/classifications/icd/Guidelines_Cause_of_Death_COVID19.pdf

3. Coronavirus disease (COVID-19) [Internet]. [cited 2021 Dec 7]. Available from: https://www.who.int/emergencies/diseases/novelcoronavirus-2019

4. Baud D, Qi X, Nielsen-Saines K, Musso D, Pomar L, Favre G. Real estimates of mortality following COVID-19 infection. Lancet Infect Dis [Internet]. 2020/03/12. 2020 Jul;20(7):773. Available from: https://pubmed.ncbi.nlm.nih.gov/32171390

5. Mokhtari T, Hassani F, Ghaffari N, Ebrahimi B, Yarahmadi A, Hassanzadeh G. COVID-19 and multiorgan failure: A narrative review on potential mechanisms. J Mol Histol [Internet]. 2020/10/04. 2020 Dec;51(6):613-28. Available from: https://pubmed.ncbi.nlm.nih.gov/33011887

6. Yang Z, Hu Q, Huang F, Xiong S, Sun Y. The prognostic value of the SOFA score in patients with COVID-19: A retrospective, observational study. Medicine (Baltimore) [Internet]. 2021 Aug 13;100(32):e26900-e26900. Available from: https://pubmed.ncbi.nlm.nih.gov/34397917

7. Vincent JL, Moreno R, Takala J, Willatts S, De Mendonça A, Bruining H, et al. The SOFA (Sepsis-related Organ Failure Assessment) score to describe organ dysfunction/failure. On behalf of the Working Group on Sepsis-Related Problems of the European Society of Intensive Care Medicine. Vol. 22, Intensive care medicine. United States; 1996. p. 707-10.

8. Wilfong EM, Lovly CM, Gillaspie EA, Huang L-C, Shyr Y, Casey JD, et al. Severity of illness scores at presentation predict ICU admission and mortality in COVID-19. J Emerg Crit care Med (Hong Kong, China) [Internet]. 2021/01/25. 2021 Jan;5:7. Available from: https://pubmed.ncbi.nlm.nih.gov/34179689

9. Evans L, Rhodes A, Alhazzani W, Antonelli M, Coopersmith CM, French C, et al. Surviving Sepsis Campaign: International Guidelines for Management of Sepsis and Septic Shock 2021. Crit Care Med [Internet]. 2021 Nov [cited 2021 Nov 17];49(11):e1063-143. Available https://journals.lww.com/ccmjournal/Fulltext/2021/11000/Surviving_Sepsis_Campaign_International.21.aspx

10. Vincent J-L, Moreno R, Takala J, Willatts S, De Mendonça A, Bruining H, et al. The SOFA (Sepsis-related Organ Failure Assessment) score to describe organ dysfunction/failure. Springer-Verlag; 1996.

11. Guan W-J, Ni Z-Y, Hu Y, Liang W-H, Ou C-Q, He J-X, et al. Clinical Characteristics of Coronavirus Disease 2019 in China. N Engl J Med. 2020 Apr;382(18):1708-20.

12. Vincent JL, de Mendonça A, Cantraine F, Moreno R, Takala J, Suter PM, et al. Use of the SOFA score to assess the incidence of organ dysfunction/failure in intensive care units: results of a multicenter, prospective study. Working group on "sepsis-related problems" of the European Society of Intensive Care Medicine. Crit Care Med. 1998 Nov;26(11):1793-800.

13. Schober P, Vetter TR. Survival Analysis and Interpretation of Time-to-Event Data: The Tortoise and the Hare. Anesth Analg [Internet]. 2018;127(3). Available https://journals.lww.com/anesthesiaanalgesia/Fulltext/2018/09000/Survival_Analysis_and_Interpretation_of.32.aspx

14. Hoffmann M, Kleine-Weber H, Schroeder S, Krüger N, Herrler T, Erichsen S, et al. SARS-CoV-2 Cell Entry Depends on ACE2 and TMPRSS2 and Is Blocked by a Clinically Proven Protease Inhibitor. Cell. 2020 Apr;181(2):271-280.e8.

15. Li XC, Zhang J, Zhuo JL. The vasoprotective axes of the renin-angiotensin system: Physiological relevance and therapeutic implications in cardiovascular, hypertensive and kidney diseases. Pharmacol Res. 2017 Nov;125(Pt A):21-38.

16. Xu Z, Shi L, Wang Y, Zhang J, Huang L, Zhang C, et al. Pathological findings of COVID-19 associated with acute respiratory distress syndrome. Vol. 8, The Lancet. Respiratory medicine. 2020. p. 420-2.

17. Trougakos IP, Stamatelopoulos K, Terpos E, Tsitsilonis OE, Aivalioti E, Paraskevis D, et al. Insights to SARS-CoV-2 life cycle, pathophysiology, and rationalized treatments that target COVID-19 clinical complications. J Biomed Sci [Internet]. 2021 Jan 12;28(1):9. Available from: https://pubmed.ncbi.nlm.nih.gov/33435929

18. Hikmet F, Méar L, Edvinsson A, Micke P, Uhlén M, Lindskog C. The protein expression profile of ACE2 in human tissues. Mol Syst Biol. $2020 \mathrm{Jul} ; 16(7): \mathrm{e} 9610$.

19. Ciceri F, Beretta L, Scandroglio AM, Colombo S, Landoni G, Ruggeri A, et al. Microvascular COVID-19 lung vessels obstructive thromboinflammatory syndrome (MicroCLOTS): an atypical acute respiratory distress syndrome working hypothesis. Crit care Resusc J Australas Acad Crit Care Med. 2020 Apr;22(2):95-7.

20. Lefrançais E, Ortiz-Muñoz G, Caudrillier A, Mallavia B, Liu F, Sayah DM, et al. The lung is a site of platelet biogenesis and a reservoir for haematopoietic progenitors. Nature. 2017 Apr;544(7648):105-9.

21. Cheng Y, Luo R, Wang K, Zhang M, Wang Z, Dong L, et al. Kidney disease is associated with in-hospital death of patients with COVID-19. Kidney Int. 2020 May;97(5):829-38.

22. Desforges M, Le Coupanec A, Dubeau P, Bourgouin A, Lajoie L, Dubé M, et al. Human Coronaviruses and Other Respiratory Viruses: Underestimated Opportunistic Pathogens of the Central Nervous System? Viruses. 2019 Dec;12(1). 